\title{
A Randomized-Controlled, Prospective Study on the Effect of Dorsal Penile Nerve Block after TURP on Catheter-Related Bladder Discomfort and Pain
}

\author{
Yunus Emre Goger ${ }^{1}$, Mehmet Serkan Ozkent ${ }^{2}$, Esra Göger ${ }^{2}$, Tansel Kılınç ${ }^{1}$, Gökhan Ecer ${ }^{1}$, \\ Mesut Pişkin ${ }^{1}$, and Atilla Erol ${ }^{1}$ \\ ${ }^{1}$ Necmettin Erbakan University Meram Medical Faculty Hospital \\ ${ }^{2}$ Konya City Hospital
}

November 3, 2020

\begin{abstract}
Purpose:In the present study, the impact of penile nerve block(PNB) on postoperative pain and CRBD in transurethral resection of prostate(TURP) patients were evaluated. Methods:Participants of the present study were selected from patients who performed TURP under spinal anesthesia for benign prostatic hyperplasia(BPH) between January 2018-July 2020. This study was planned as a single center, randomized-controlled prospective study. The patients were divided into two groups; Group 1 was administered Control(n:40), and Group 2 ultrasonography(USG) guided PNB(n:40). The patients were included in the Groups respectively. Visual analogue scale(VAS) scores were questioned and recorded in order to evaluate the postoperative pain complaints of the patients after the operation. In addition, in order to evaluate the CRBD, VAS scores were questioned and recorded as 0-1th hour, 1st-2nd hours, 2nd - 4th hours, 4th-8th hours, 8th-12th hours, and 12th-24th hours. In addition, postoperative pain and analgesic need were recorded. Tramodol was given to patients with moderate to severe CRBD. The findings was compared between to the Groups. Results:There was no statistical difference between Group 1 and Group 2 between demographic and per-operative data. The CRBD and pain-related VAS scores was significantly higher in Group 1 between the 0-8th hours. There was no difference between VAS scores in the postoperative 8-24th hours. In total 24 hours, Group 2's need for tramodol for CRBD and pain was significantly less than Group 1. On examining the factors affecting CRBD in the multivariate analysis, age, body mass index(BMI), prostate volume, operation time do not affect CRBD statistically, only PNB reduces CRBD (p: 0.029). While less drug-related complications were observed in Group 2, no serious complications related to PNB were observed. Conclusion:PNB is the effective method for the decrease pain and CRBD after urological surgery.It will also reduce the need for analgesics, and provide the painless patients in postoperative period.
\end{abstract}

A Randomized-Controlled, Prospective Study on the Effect of Dorsal Penile Nerve Block after TURP on Catheter-Related Bladder Discomfort and PainYunus Emre Göger ${ }^{1}$, Mehmet Serkan Özkent $^{2}$, Esra Göger ${ }^{3}$, Muzaffer Tansel Kılınç ${ }^{1}$, Gökhan Ecer ${ }^{1}$, Mehmet Mesut Pişkin ${ }^{1}$, Atilla Erol ${ }^{4}$

\section{Department of Urology, Meram Medical Faculty, Necmettin Er- bakan University, Konya, Turkey.}

2- Department of Urology, Konya City Hospital, Konya, Turkey. ${ }^{3}$ - Department of Anaesthesiology, Konya City Hospital, Konya, Turkey. ${ }^{4}$ - Department of Anaesthesiology, Meram Medical Faculty, Necmettin Erbakan University, Konya, Turkey.Running Title: Dorsal Penile Nerve Block after TURPKey Words: Postoperative pain, penile nerve block, urological surgery, anesthesiaArticle Type: Original ArticleNumber of 
tables: 6Number of figures: Abstract word count: 300Manuscript word count: 3479Number of references: 43

Corresponding author: Mehmet Serkan Ozkent, MDDepartment of Urology, Konya City Hospital, Konya, Turkey.Adress: Akabe, 42020 Karatay/KonyaPhone: +90 50593641 55Email: msozkent@gmail.comORCID ID: https://orcid.org/0000-0002-6613-0671Web of Science Researcher ID: AAJ-9851-2020Yunus Emre Goger, Assistant Prof.Department of Urology, Meram Medical Faculty, Necmettin Erbakan University, Konya, Turkey.Phone: +90 53341507 53Email: dr_yegoger@yahoo.comORCID ID: https://orcid.org/0000-0002-4480-9093Esra Goger, MD.Department of Anaesthesiology, Konya City Hospital, Konya, Turkey.Phone: $\quad+90 \quad 535 \quad 933 \quad 74$ 29Email: dr.esragoger@gmail.comORCID ID: https://orcid.org/0000-0002-3237-8695

Muzaffer Tansel Kılınc, MD.

Department of Urology, Meram Medical Faculty, Necmettin Erbakan University, Konya, Turkey.Phone: +90 55369414 28Email: m.tanselkilinc@gmail.comORCID ID: https://orcid.org/0000-0002-10243394Gokhan Ecer, MD.Department of Urology, Meram Medical Faculty, Necmettin Erbakan University, Konya, Turkey.Phone: $+90530 \quad 863 \quad 06$ 37Email: ecergokhan@gmail.comORCID ID: https://orcid.org/0000-0002-2805-8664Mehmet Mesut Piskin, Professor.Department of Urology, Meram Medical Faculty, Necmettin Erbakan University, Konya, Turkey.Phone: +90 53767110 74Email: drmesutpiskin@yahoo.comORCID ID: https://orcid.org/0000-0002-0528-6699Atilla Erol, Professor.Department of Anaesthesiology, Meram Medical Faculty, Necmettin Erbakan University, Konya, Turkey.Phone: +90532 76739 38Email: atillaerol@yahoo.comORCID ID: https://orcid.org/0000-0002-2376-2759

DeclarationsWe have agreed to the submission and that the article is not currently being considered for publication by any other print or electronic journal. We certify that none of the paper's contents have been previously published.Ethical ApprovalAll procedures performed in studies involving human participants were in accordance with the ethical standards of the institutional and/or national research committee and with the 1964 Helsinki declaration and its later amendments or comparable ethical standards. The analysis and data collection were performed following the after written informed consent was obtained from all patients. The institutional human research ethics committee approved the protocol 2020/ (Necmettin Erbakan University, Meram Medical Faculty Ethics Committee).FundingThe authors declare that this work has not received any funding. Competing interestsThe authors declare that they have no competing interests.Availability of data and materialData are available on request.Code availabilityNot applicable.AcknowledgementsNot applicable.Authors' contributionsYE Goger: Protocol/project development; Data collection or management; Manuscript writing/editing; Critical revision of the manuscript; Supervision; Approval of the final manuscriptMS Ozkent: Protocol/project development; Data collection or management; Data analysis; Manuscript writing/editing; Critical revision of the manuscript; Supervision; Approval of the final manuscriptE Göger: Data collection or management; Manuscript writing/editing; Critical revision of the manuscript; Administrative, technical, or material support; Approval of the final manuscriptMT Kilinc: Data collection or management; Manuscript writing/editing; Critical revision of the manuscript; Administrative, technical, or material support; Approval of the final manuscriptG Ecer: Data collection or management; Manuscript writing/editing; Critical revision of the manuscript; Administrative, technical, or material support; Approval of the final manuscriptMM Piskin: Protocol/project development; Data collection or management; Data analysis; Manuscript writing/editing; Critical revision of the manuscript; Approval of the final manuscriptA Erol: Protocol/project development; Data collection or management; Data analysis; Manuscript writing/editing; Critical revision of the manuscript; Approval of the final manuscript

\section{Hosted file}

Main Text.pdf available at https://authorea.com/users/343046/articles/490491-a-randomizedcontrolled-prospective-study-on-the-effect-of-dorsal-penile-nerve-block-after-turp-oncatheter-related-bladder-discomfort-and-pain 


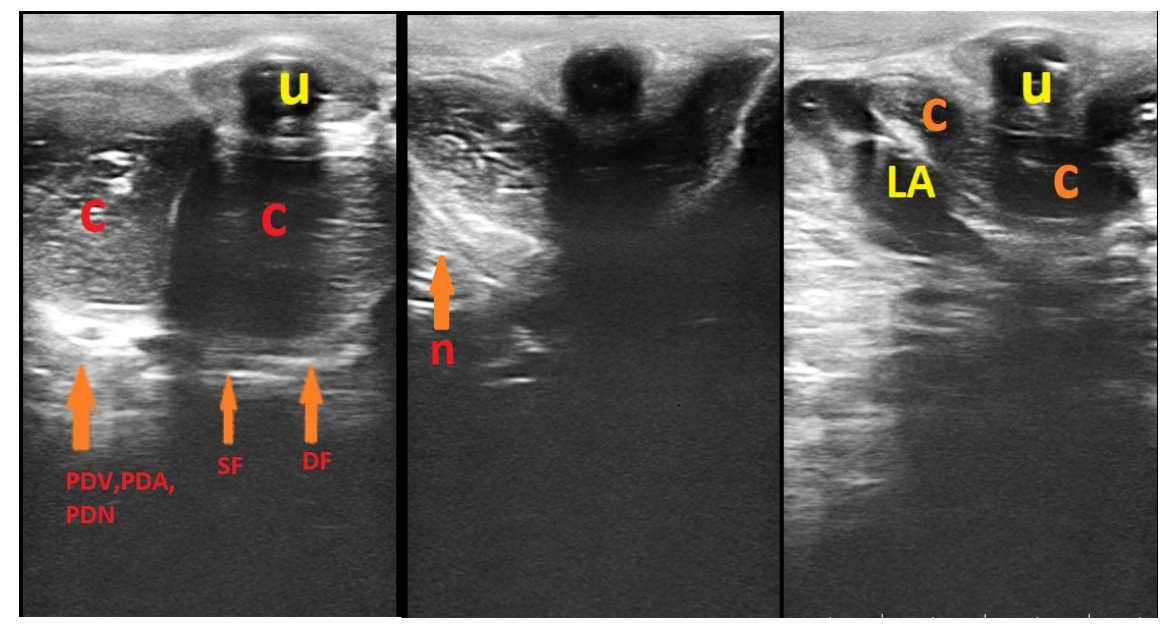

